\title{
A SYSTEM OF GALACTIC CONSTANTS BASED ON STELLAR DYNAMICS
}

\author{
S. NINKOVIĆ \\ Astronomska opservatorija \\ Volgina 7 \\ 11050 Beograd, Yugoslavia
}

\begin{abstract}
A system of galactic constants is proposed. Due to the intention of basing it on stellar dynamics, the working hypothesis is the assumption that the Galaxy is in a steady state characterized with axial symmetry. Then, as easy to see, one reaches a system containing seven independent constants typical for the present assumption. The current knowledge concerning the amounts of these constants is discussed.
\end{abstract}

\section{Introduction}

Among the questions where the contribution of J. H. Oort appears as essential is, certainly, the one concerning the galactic constants. The dynamical constants have been considered by the present author in an earlier paper of his (Ninković, 1987). This time a system of constants based on a given dynamical approach (steady state and axial symmetry) should be formed.

\section{Formation of the System of Constants}

In principle, any quantity of galactic astronomy taken at the position of the Sun may be considered as a "constant". With regard to such a variety of possibilities it is necessary to limit the scope to some kind of quantities. It is clear that the quantities concerning the spatial distribution and kinematics of galactic objects are of interest to galactic dynamics, though strictly speaking they have no dynamical nature since they do not incorporate the notion of force. On the other hand the number of necessary constants and their choice depend on the assumed dynamical approach. For example, if 
the Galaxy were spherically symmetric, then any plane connecting the Sun and the galactic center could be chosen as the plane of galactic equator, but three constants would be also necessary for transformation from the independent equatorial system to the galactic coordinates as it is the case now. In the same way the residual motion of the Sun would require three quantities to be known irrespectively of the symmetry.

Throughout this contribution it will be assumed that the Galaxy is characterized with steady state and axial symmetry. In that case, as easy to see, only two quantities are enough to describe the galactocentric position of the Sun - its distance to rotation axis and that to the galactic plane; these are the first two constants of the present system. The next will be the velocity of the local centroid, i.e. the local velocity of galactic rotation in view of the upper assumption.

These quantities, though essentially not dynamical, but important to the galactic dynamics, are followed by a group of purely dynamical quantities - the (gravitational) potential and its derivatives. As usually done, only the derivatives up to the second order are considered. Thus in the case of steady state and axial symmetry, four constants appear - the local values of the potential, itself, of the first and second radial derivatives and of the second vertical derivative. In this way one obtains a system of galactic constants, containing seven constants, based on stellar dynamics and useful for its purposes.

TABLE 1. The System of Galactic Constants

\begin{tabular}{ll}
\hline$R_{\odot}$ & $\begin{array}{l}\text { distance of the Sun to the rotation axis; } \\
Z_{\odot}\end{array}$ \\
$u_{\odot}$ & $\begin{array}{l}\text { the Sun's vertical coordinate; } \\
\text { the rotation velocity of the galactic disc } \\
\text { (the centroid containing the Sun); }\end{array}$ \\
$\Pi_{\odot}$ & the galactic potential; \\
$\left(\frac{\partial \Pi}{\partial R}\right)_{\odot}\left(\frac{\partial^{2} \Pi}{\partial R^{2}}\right)_{\odot}\left(\frac{\partial^{2} \Pi}{\partial Z^{2}}\right)_{\odot}$ & \\
\hline
\end{tabular}

The latter five quantities are taken in the galactic plane at the distance $R_{\odot}$ from the galactic center; in the case of the latter three the explanations are self-evident.

\section{The Actual Situation with the Constants Values}

As well known, the IAU since almost ten years has been recommending the value of $8.5 \mathrm{kpc}$ for $R_{\odot}$. As for $Z_{\odot}$, though there is no official recommendation, it is very well known that $\left|Z_{\odot}\right|<<R_{\odot}$ (usually thought that $Z_{\odot}$ 
is between $+10 p c$ and $+20 p c$ ) which justifies the consideration of the other quantities mentioned above in the galactic plane instead of exactly at the Sun. The value of the local rotation velocity depends on the one of the local asymmetric drift which seems to be about $14 \mathrm{kms}^{-1}$ (e. g. Ninković, 1992). The value of the galactic potential is expressed through that of the local escape velocity which seems to be significantly higher than thought earlier, perhaps even $600 \mathrm{kms}^{-1}$, or more (e. g. Ninković, 1994). The first radial derivative of the potential is expressed through the corresponding value of the circular velocity where, as well known, the IAU has been recommending $220 \mathrm{kms}^{-1}$. The second derivative can be found through the slope of the circular velocity, or through the ratio of the Oort constants, i. e. through the ratio of the local velocity dispersions (in the galactic plane) for the disc. The results seem to be discordant (e.g. Marochnik and Suchkov, 1984 - p. 235 ), but the disagreement can be removed so that an agreement between the locally flat rotation curve and the usual value, of about 1.6 , for the velocity dispersion ratio results (Ninković, 1992). The value of the second vertical derivative depends on the problem of the local density. Since at present it is unclear how high the percentage of the local dark matter is, this question cannot be answered surely.

\section{Conclusion}

As a conclusion one should emphasize that the galactic constants in the present paper are considered in the framework of a system finding its basis in a given dynamical assumption. It is understandable that the next step should be the verification of the given dynamical assumption which may result in a new assumption involving additional quantities locally considered as constants. Also, one should be aware that in the actual calculations yielding the values of the constants are used samples of concrete stars where a correlation between their physical and kinematical properties, having no dynamical origin, may be of importance for the final results so that the working dynamical hypothesis may seem doubtful.

\section{References}

Marochnik, L. S. and Suchkov, A. A.:1984, Galaktika, "Nauka", glav. red. fiz.-mat. lit., Moskva.

Ninković, S.:1987, Publ. Astron. Inst. Czech. Acad. Sci., 69, 317.

Ninković, S.:1992, Astrophys. Space Sci., 187, 159.

Ninković, S.:1994, IAU Symp. 165, in press. 